\title{
Qualitative and Quantitative Techniques for Differential Equations Arising in Mathematical Physics
}

\author{
Rehana Naz, ${ }^{1}$ Mariano Torrisi, ${ }^{2}$ Igor Leite Freire, ${ }^{3}$ and Imran Naeem ${ }^{4}$ \\ ${ }^{1}$ Centre for Mathematics and Statistical Sciences, Lahore School of Economics, Lahore 53200, Pakistan \\ ${ }^{2}$ Dipartimento di Matematica ed Informatica, Università di Catania, Viale A. Doria 6, 95125 Catania, Italy \\ ${ }^{3}$ Centro de Matemática, Computação e Cognição, Universidade Federal do ABC, Santo André, Brazil \\ ${ }^{4}$ Department of Mathematics, School of Science and Engineering, Lahore University of Management Sciences, Lahore 54792, Pakistan \\ Correspondence should be addressed to Rehana Naz; drrehana@lahoreschool.edu.pk
}

Received 19 November 2017; Accepted 19 November 2017; Published 26 December 2017

Copyright (C) 2017 Rehana Naz et al. This is an open access article distributed under the Creative Commons Attribution License, which permits unrestricted use, distribution, and reproduction in any medium, provided the original work is properly cited.

Differential equations are a key tool in modeling physical phenomena. Most of physical laws of natural sciences are expressed in terms of differential equations, for example, balance laws of mass or energy and momentum. Additionally, differential equations are also employed in modeling population dynamics, diseases, and others.

In this special issue we focus on bringing together applications and theoretical developments of differential equations oriented to problems arising in physical sciences. To this end, this issue will provide a forum to investigate the advances in the qualitative and quantitative techniques for ordinary differential equations, partial differential equations, fractional differential equations, integrodifferential equations, and difference equations.

The potential topics of this special issue include symmetries, differential equations, and applications; optimal control; equivalence transformations and classical and nonclassical symmetries; reduction techniques and solutions and linearization; conserved quantities in natural phenomena; completely integrable equations in mathematical physics; recursion operators, infinite hierarchy of symmetries, and/or conservation laws; equations admitting weak soliton solutions; models for air pollution and underground pollution; mathematical methods for extended thermodynamics; numerical techniques for problems arising in the modeling of physical process; ad hoc methods for solutions.

The response to this special issue was beyond our expectation. We received 53 papers to consider for publication, which fall in different areas of the above-mentioned research fields. All manuscripts submitted to this special issue went through a rigorous refereeing process. Based on the reviewers' reports, 8 original research articles finally have been accepted for publication. In the following, we briefly describe the significance of the key contributions to this special issue.

In "Numerical Simulation to Air Pollution Emission Control near an Industrial Zone" P. Oyjinda and N. Pochai propose an atmospheric diffusion model to describe the released air pollutant concentration by an industrial plant. An explicit finite difference technique is employed to approximate the air pollutant concentration. The authors suggest that the air pollutant concentration levels for each monitoring point are controlled to be at or below the national air quality standard near industrial zone index.

In "Numerical Solutions of Coupled Systems of Fractional Order Partial Differential Equations" Y. Li and K. Shah developed a systematic numerical technique by utilizing shifted Legendre polynomials to construct approximate solutions to a class of coupled systems of fractional order partial differential equations. With the aid of operational matrices of fractional order integrations and differentiations, the system under consideration is converted to a system of easily solvable algebraic equation of Sylvester type. The established technique is demonstrated by some test problems, and error analysis is carried out as well.

$\mathrm{X}$. Jiang and X. Wang in the article "Global Well-Posedness for a Class of Kirchhoff Type Wave System" investigate the well-posedness of the Kirchhoff type wave system with nonlinear source, dissipative and viscoelastic terms, subject 
to the big initial boundary conditions. For the subcritical case, the global solutions exist provided the initial value belongs to the stable set, while the finite time blowup occurs for the case when initial value belongs to the unstable set. For the supercritical case, the corresponding solution blows up in a finite time under some given conditions on the initial values.

In "Geometrical/Physical Interpretation of the Conserved Quantities Corresponding to Noether Symmetries of Plane Symmetric Space-Times" B. Jamil et al. investigate Noether symmetries of geodetic Lagrangians of plane symmetric spacetimes and their corresponding conserved quantities. They provide the number of Noether symmetries that plane symmetric spacetimes can admit. In particular, the largest number of Noether symmetries is achieved for the Minkowski spacetime.

In "Heat Transfer in a Porous Radial Fin: Analysis of Numerically Obtained Solutions" R. Jooma and C. Harley study a model describing heat transfer in a porous radial fin from numerical point of view. Namely, the authors employ the Crank-Nicolson method with and without the NewtonRaphson predictor-corrector to perform their analysis. Semianalytical steady state solutions are obtained via differential transformation method. Comparisons between the results obtained in this paper and other recent reported ones in the literature are also presented.

In "An Iterative Method for Solving of Coupled Equations for Conductive-Radiative Heat Transfer in Dielectric Layers" by V. Chekurin and Y. Boychuk, the authors consider a mathematical model to describe a combined conductive-radiative heat transfer in a dielectric layer, which emits, absorbs, and scatters IR radiation both in its volume and on the boundary. A nonlinear stationary boundary-value problem for coupled heat and radiation transfer equations for the layer, which exchanges energy with external medium by convection and radiation, has been formulated. In the case of optically thick layer, when its thickness is much more of photon-free path, the problem becomes the singularly perturbed one. In the inverse case of optically thin layer, the problem is regularly perturbed and it becomes the regular (unperturbed) one when the layer's thickness is of order of several photon-free paths. An iterative method to solve the unperturbed problem has been developed and its convergence has been tested. By using this method the temperature field and radiation fluxes have been studied.

In "A Fast Implicit Finite Difference Method for Fractional Advection-Dispersion Equations with Fractional Derivative Boundary Conditions" T. Liu and M. Hou consider the fractional advection-dispersion equations modeling the transport of passive tracers carried by fluid flow in a porous medium. For these equations with fractional derivative boundary conditions, the authors develop an implicit finite difference method. First-order consistency, solvability, unconditional stability, and first-order convergence of the method are proved. Then, they show a fast iterative method for the implicit finite difference scheme, which only requires a storage of $O(K)$ and a computational cost of $O(K \log K)$. Usually, the Gaussian elimination method requires a storage of $O\left(K^{2}\right)$ and a computational cost of $O\left(K^{3}\right)$.
In their paper "Canonical Forms and Their Integrability for Systems of Three 2nd-Order ODEs" S. Zahida et al. construct the differential invariants and their corresponding canonical forms for systems of three 2nd-order ODEs possessing three-dimensional Lie algebras. The extension up to $k$ th order system of three 2nd-order ODEs is derived. Moreover, singularity in invariant structure for the canonical forms is investigated and the integrability of these canonical forms is discussed. Some physical examples from system of particles mechanics are shown.

\section{Acknowledgments}

We thank all the authors for their valuable contributions to this special issue. We thank the honorable reviewers for their time, efforts, and cooperation to review all the submitted articles.

Rehana Naz

Mariano Torrisi Igor Leite Freire Imran Naeem 


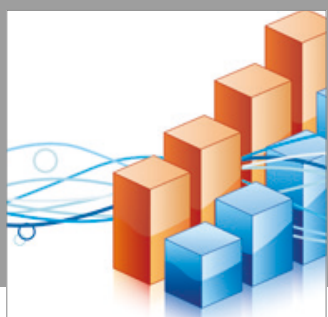

Advances in

Operations Research

vatersals

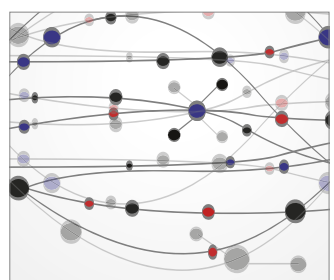

\section{The Scientific} World Journal
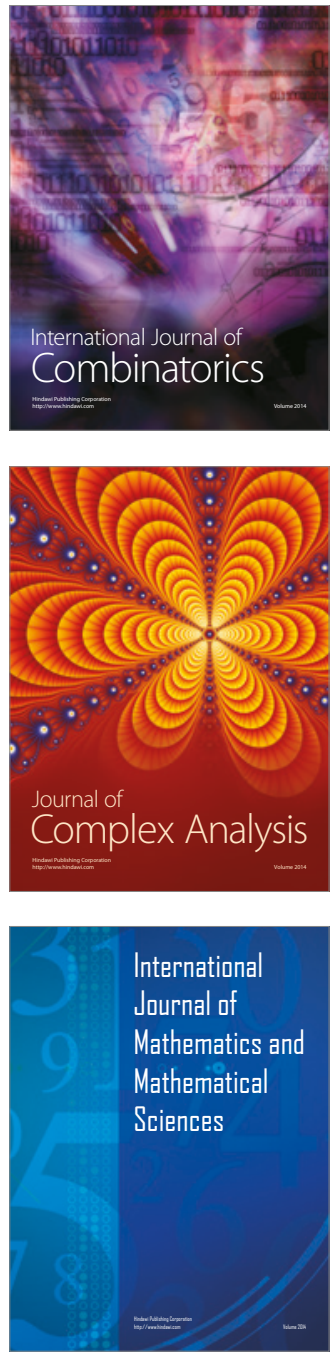
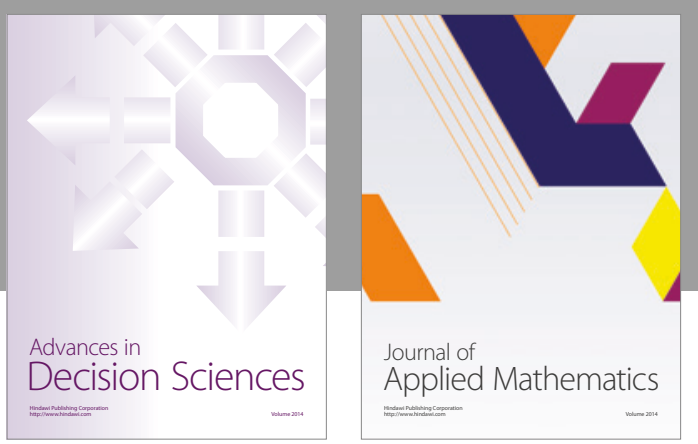

Algebra

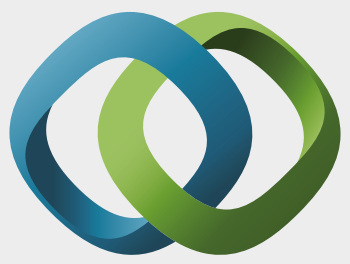

\section{Hindawi}

Submit your manuscripts at

https://www.hindawi.com
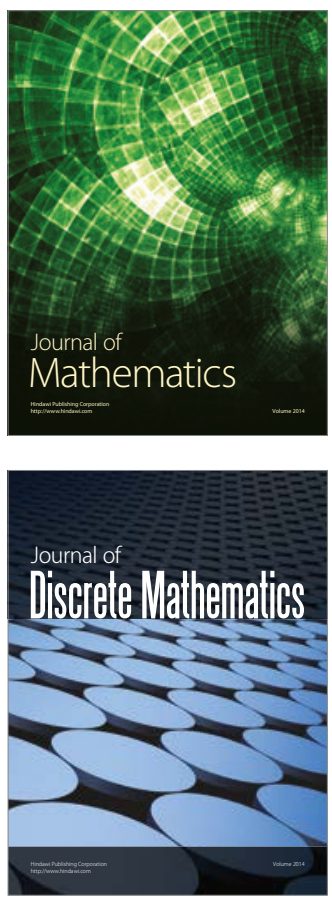

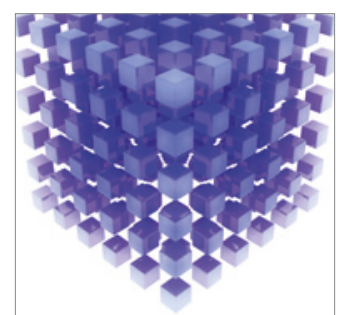

Mathematical Problems in Engineering
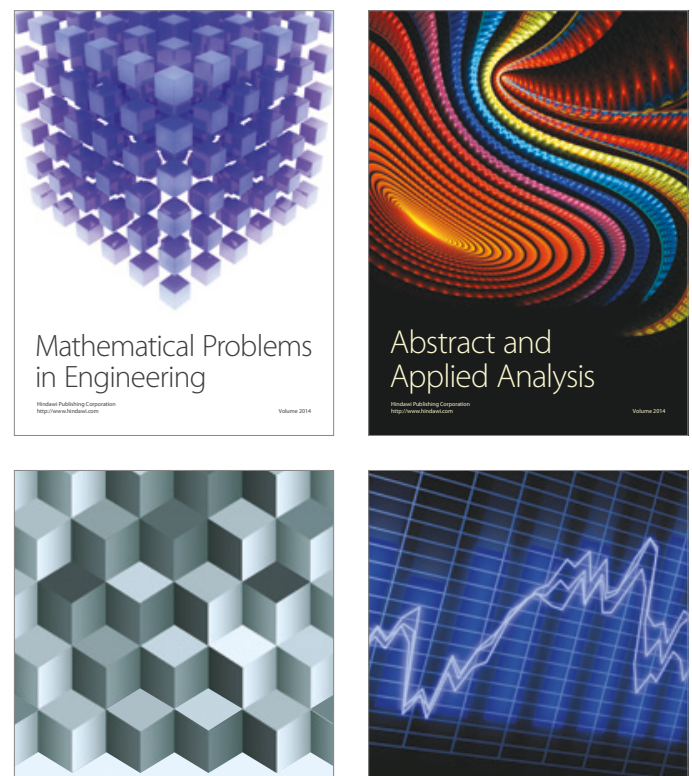

Journal of

Function Spaces

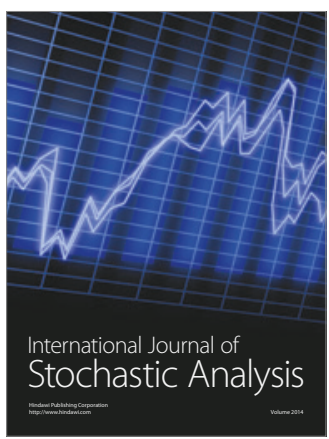

Probability and Statistics
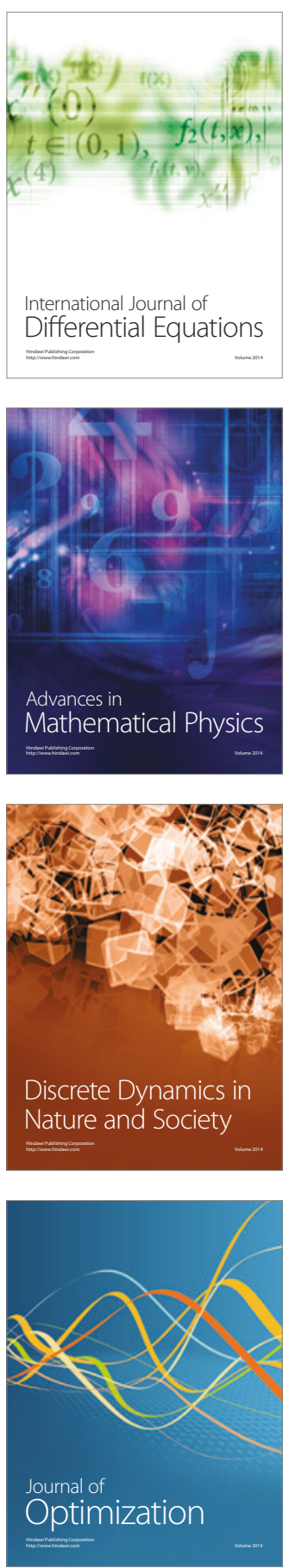